\title{
Un regard oblique : «L'œil du sorcier » dans Une vie de boy de Ferdinand Oyono
}

\author{
Pierre Vaucher, Université Laval
}

\section{Introduction}

Dans un numéro de Notre Librairie consacré à la question de la violence dans «les littératures francophones du Sud », le critique Xavier Garnier affirme ceci :

Le problème de tous ces romanciers, qui cherchent à violenter le monde par leurs textes, n'est pas de mettre en forme tel ou tel récit, mais d'énoncer un récit qui soit une forme. Le monde regorge de récits informes qui rentrent sagement dans des cases déjà prévues pour eux, ces récits peuvent raconter les pires violences, ils resteront aussi inoffensifs que des limaces. Toute la difficulté, et tout l'art, consiste à forger des récits aux formes dures, des récits parfois naïfs, mais nécessaires et urgents comme des récits d'enfants (6).

Textes qui violentent le monde, récits qui sont des formes («récits-formes », pourrait-on dire), «récits aux formes dures »: les formules de Garnier ont l'intérêt de souligner le rapport réciproque entre le social et l'espace du langage (l'un ne pouvant être simplement le reflet de l'autre); l'articulation toujours renouvelée entre texte et hors-texte. À l'opposé d'une forme indifférente, qui «accueillerait» son objet ou qui se superposerait à lui pour en épouser les contours aussi fidèlement que possible, il y aurait une forme qui se pense dans sa relation au monde, et, se faisant, détermine son contenu.

C'est en repartant de cette idée-force d'une "violence du texte », d'un texte qui tente d'imposer sa forme au monde, que nous voudrions réinterroger le motif du regard dans Une vie de boy de Ferdinand Oyono. Ce thème a déjà été traité à plusieurs reprises par les critiques, mais sans toutefois montrer à quel point le regard se situe à la jonction entre l'esthétique de l'œuvre et son contenu.

Kwabena Britwum, dans « Regard, mémoire, témoignage : ou l'œil du sorcier dans “Une vie de boy" de Ferdinand Oyono » (1977), est sans doute le premier à souligner l'importance du regard. Chez lui, l'œil, synecdoque du témoin oculaire, est envisagé comme "instrument de témoignage » (37). Mais le critique insiste surtout sur sa force persuasive : il s'agit d'un « regard remémorant» (38) doté d'une mémoire et d'une intention qui, ironiquement, donne à voir le présent à la lumière du passé. Arthur Flannigan, dans " "The Eye of the Witch" : Non-Verbal Communication and the Exercise of Power in Une Vie de Boy» (1982), explore les rapports entre 
regard et pouvoir. Le roman d'Oyono mettrait en cause un conflit implicite entre dominants (colons) et dominés (colonisés) qui peut être retracé à travers les jeux de (non-)regards qui s'établissent entre eux. Suivant de près ce fil analytique, Flannigan tente de situer dans le récit le point critique où s'opèrent une prise de conscience et un renversement des rapports de force. Jacques Bourgeacq, dans « The Eye Motif and Narrative Strategy in Ferdinand Oyono's Une Vie de boy: An Ethno-Cultural Perspective » (1993), adopte une approche culturaliste, mettant en rapport le regard avec le concept de «Djambe », en référence au contexte de la société Maka du Sud du Cameroun. L'article a l'intérêt de souligner la dimension implicite de ce regard, qui traverse les apparences pour atteindre l'invisible. Enfin, Simon Amegbleame, dans « Le regard du boy et la conscience du commandant : une lecture d'Une vie de boy de Ferdinand Oyono » (2004), établit une équivalence entre la caméra et le regard, enregistrant les moindres faits et gestes des personnages.

Rappelons que l'année 1956 (lorsqu'Oyono publie son roman) correspond à un tournant décisif dans l'histoire du continent africain : c'est la période de la décolonisation. La tension entre la France et les colonies atteint un paroxysme. Bien que la chute de l'empire colonial soit programmée, la Métropole veut à tout prix préserver son hégémonie. Malgré les ignominies commises (on pense notamment à la répression sanglante de 1955 contre la population camerounaise), la bonne conscience coloniale continue à faire foi ${ }^{1}$.

Or, dans Une vie de boy, c'est le regard qui donne forme à cette réalité des colonies. Il permet de lier une manière de dire, touchant alors à la singularité d'un texte, et un discours sur le monde. Au discours social, dominé par quelques-unes des figures-clés de l'univers colonial, Oyono superpose ce que l'on pourrait appeler un « discours du regard », celui de Toundi Ondoua, à partir duquel les « Blancs » ainsi que les « Noirs » vont être entièrement mis à nu. C'est à ce niveau qu'opère la violence du texte : le regard de l'enfant, parce qu'il n'est freiné par aucun interdit et enregistre tout, en particulier ce qui devrait passer inaperçu, devient un prodigieux moyen de dérision. À travers lui, en effet, le monde colonial et tout l'appareil idéologique qui assure son maintien se dégonflent comme un ballon de baudruche.

Le roman retrace le parcours de Toundi sous la colonisation française. Selon un schéma bien classique, le récit se veut la traduction de son journal intime récupéré par un témoin anonyme alors que le personnage est sur le point de mourir. Trois étapes jalonnent sa progression qui sont autant de divorces significatifs. Dans un premier temps, Toundi se met au service du 
père Gilbert. Il entreprend alors d'écrire un journal, rapportant de façon naïve les événements de son quotidien. Puis, il est engagé comme boy par le commandant de cercle établi à Dangan, dont la femme entretient une relation adultère avec le régisseur de prison M. Moreau. Sous le coup d'une désillusion progressive, il découvre ainsi la déchéance de ses maîtres. Toutefois, ceux-ci ne supportent plus d'être observés par lui, un simple boy. En effet, la persistance de son regard répercute sur eux l'image, inadmissible, de leur échec et de leur mauvaise conscience. Ils décident donc de se défaire de ce témoin gênant en l'accusant à tort de complicité de vol. Dans la dernière étape de son parcours, Toundi prendra la fuite en Guinée espagnole en emportant avec lui son journal.

On voit ainsi comment un regard informe, une sorte de « caméra vérité » qui enregistrerait machinalement le réel, se transmue en un regard qui, par son pouvoir de suggestion, devient une instance récriminatoire. L'originalité de notre approche tient au fait qu'elle montre comment le regard, chez Oyono, étant situé à la confluence entre le dire et le dit, devient un puissant procédé argumentatif. Grâce à celui-ci, la critique implicite du système colonial parvient à masquer sa rhétorique, faisant comme si le discours qui la sous-tend émanait de la représentation elle-même.

\section{Regard et représentation du pouvoir}

Dans Une vie de boy, le regard fait l'objet d'une appropriation subversive : il est un moyen de libération. Si cette émancipation ne s'accomplit pas de fait (l'échappée de Toundi en Guinées espagnole prend fin avec la mort du fuyard), elle n'en est pas moins en perspective, comme un point qui se dessine à l'horizon : le journal du boy sera récupéré par un " compatriote », de sorte que le témoignage survit au témoin lui-même. Pourquoi le regard? Sa force provient de son caractère labile, « rusé ». Par rapport au discours, il est moins contrôlable, plus insidieux, agissant par le silence. Plus qu'un instrument, c'est un vecteur : non pas agi, il est ce qui permet d'agir, d'inciter un changement d'état, de donner forme. Son indétermination en fait un site privilégié de transgression qui se produit autant sur le plan social (il a, comme on vient de l'évoquer, un rôle émancipatoire) qu'au niveau formel (il est la marque d'un style). Arrêtons-nous sur le premier de ces deux aspects.

$\mathrm{Si}$, dans le roman d'Oyono, le regard revêt une telle importance, c'est d'abord parce qu'il permet une représentation du pouvoir. Il fonctionne en effet comme un code implicite qui contribue, au-delà du verbal, à régler les rapports entre les personnages et à définir leur place au 
sein d'une hiérarchie rigide. Comme Flannigan le souligne dans son article (53), le regard constitue une forme de contrôle exercé par l'autorité coloniale sur la population locale. Il est l'apanage exclusif des colons, qui se réservent le droit d'exercer certains types de regard sur les colonisés qui, pour leur part, leur sont prohibés.

Lorsque Toundi est convoqué par le commandant de Cercle de Dangan pour subir son « examen d'entrée » comme boy, les rapports hiérarchiques sont définis et signifiés, avant même tout échange de mots, par la pesanteur du voir : «Après m'avoir longuement observé, mon nouveau maître me demande à brûle-pourpoint si j'étais un voleur » (Oyono 33) ; « J'allais sortir le petit livre de prières de la poche arrière de mon short quand le commandant arrêta mon geste d'un signe. Il me regarda un moment à travers les volutes de fumée qu'il me soufflait au visage. Il s'assit. Je baissai la tête. Je sentais son regard sur mon front (ibid.) ». Toundi ne semble pas remettre en cause cet œil scrutateur. Pourtant, par l'examen qu'il cautionne et son caractère irrévocable, celui-ci définit les places de dominant/dominé, de même qu'il donne à l'échange une aura de fatalité découlant de l'impression de suspicion. En effet, reflet du racisme propre à l'idéologie coloniale, la persistance du regard du commandant convoque l'image stéréotypée du « Noir » comme être déchu et immoral, et que la discussion sur l'enfer qui suit immédiatement vient d'ailleurs renforcer. Il est à un tel point chargé de significations qu'il devient pour Toundi sensible au-delà même du visible («Je baissai la tête. Je sentais son regard sur mon front (ibid.) »).

C'est justement parce que le regard fait sens qu'il est refusé aux membres de la communauté noire. Cette prohibition visuelle vise en particulier le personnel de la Résidence du commandant qui entretient des rapports particulièrement tendus avec les colons, étant donné son accès direct à leur sphère privée. Les domestiques vivent le regard comme une dépossession, une dénégation, qui apparaît clairement lorsque le garde veut informer ses compatriotes de l'adultère de la femme du commandant avec le régisseur de la prison.

Tandis qu'il ne parle que par métaphores, son témoignage est l'objet d'une double négation : «- En vérité, avait-il commencé, dans un souffle, on ne peut pas dire que je n'aie pas vu le régisseur de la prison prendre congé de Madame après minuit... (95) » [je souligne]. Si cette double négation équivaut à une affirmation, elle permet surtout de préserver, dans l'espace de l'énonciation, la négation du voir. L'acte de vision est ainsi neutralisé, rendu passif; il est vécu comme quelque chose d'extérieur à soi : «Si je parle, c'est parce que j'ai une bouche. Si je 
vois, c'est parce que j'ai des yeux. L'œil va plus loin et plus vite que la bouche, rien ne l'arrête dans son voyage... (ibid.) ». C'est que le regard, dans le roman, est inséparable du jugement; il rend possible le commentaire, la critique. Il constitue donc un enjeu de pouvoir : voir, c'est être en mesure d'affaiblir l'Autre. Nier le regard, c'est reconnaître sa charge subversive, potentiellement dangereuse.

Parmi tous ses concitoyens, Toundi est le seul qui rompt ostensiblement l'interdiction du « regard jugeant » imposée par l'élite européenne. Toutefois, cette transgression ne découle pas à première vue d'un mouvement de révolte. Ainsi, dès le départ, toutes les actions de Toundi sont ramenées à sa soi-disant " gourmandise ». Des petits cubes sucrés lancés aux enfants par le père Gilbert à la tenue d'un journal personnel, Toundi est appâté par l'univers des «Blancs » et ses agréments, occasionnant la colère puis le rejet de son père. Il prend goût à tout. Ce « vice », tel qu'il le caractérise, lui vaut l'exclusion familiale et celle de sa communauté. Niant quelque part ce qu'il est devenu pour le colon, c'est à cette prétendue "gourmandise » qu'il rapporte sa curiosité incoercible qui causera sa perte : "Ma mère me disait toujours que ma gourmandise me conduirait loin. Si j'avais pu prévoir qu'elle me conduirait au cimetière... Elle avait raison, ma pauvre mère... (13)».

Pourtant, au fur et à mesure que le récit progresse, son regard insistant tend à refléter une forme d'émancipation. Ainsi, ce n'est pas sans un certain soulagement qu'il assiste à l'humiliation de ses maitres. Découvrant pour la première fois la nudité du commandant, il avoue : «Cette découverte m’a beaucoup soulagé. Cela a tué quelque chose en moi... Je sens que le commandant ne me fait plus peur. Quand il m'a appelé pour que je lui donne ses sandales, sa voix m'a paru lointaine, il m'a semblé que je l'entendais pour la première fois. Je me suis demandé pourquoi j'avais tremblé devant lui » (45). Si l'observation est encore, à ce niveau, fortuite, elle sera par la suite provoquée, notamment une fois qu'il se délecte de la conduite adultère de la femme de son maître. Toute l'astuce d'Oyono consiste à donner à voir cette émancipation comme si elle suivait un cours naturel, inéluctable.

Kalisia, la nouvelle femme de chambre, rapporte ce regard à un savoir jugeant : "C'est bête, mais avec les Blancs c'est comme ça... Pour lui tu seras... je ne sais comment appeler ça... tu seras quelque chose comme l'œil du sorcier, qui voit et qui sait. Un voleur ou quelqu'un qui a quelque chose à se reprocher ne peut jamais se sentir tranquille devant cet œil-là... » (152). 


\section{D'un regard excessif à une écriture allusive}

La transgression du regard de Toundi se confond avec une écriture insinuante qui dérange l'espace du dicible. Bien plus qu'une «illusion référentielle $»^{2}$, qui confère son authenticité au texte, la scénographie du journal intime permet d'introduire un discours incisif s'imposant avec d'autant plus de persuasion qu'il fait mine de se renier en tant que tel. Et si l'œuvre d'Oyono ne cesse de renvoyer à la situation coloniale des années cinquante, elle n'en apparaît jamais comme une dénonciation explicite, de même qu'elle se dérobe à l'explication réaliste'

Les velléités d'ascension sociale de Toundi attirent ainsi l'attention sur le contrat colonial par lequel la France justifiait sa mainmise sur les colonies africaines. Cet accord tacite, il faut le rappeler, stipulait l'élévation morale du colonisé, l'amélioration de son niveau de vie et une vague promesse d'égalité, en échange de sa «collaboration» inconditionnelle, c'est-à-dire sa soumission à l'autorité (Corcoran 20).

Or, Oyono entend amener son lecteur à prendre conscience du caractère frauduleux de ce contrat. Pour ce faire, il donne au colon blanc la figure d'un être immoral et vicieux, à l'opposé du modèle de civilisation que celui-ci préconise et que suggère, dans le roman, l'euphémisme « apprendre à vivre » annonçant la bastonnade à mort de deux individus soupçonnés d'avoir volé (Oyono 114). Mais plutôt que de revêtir un caractère trop manifeste, cette représentation de la déchéance du «Blanc» apparaît de manière indicielle. De la sorte, elle semble aller de soi, puisque c'est le lecteur, en dernier ressort, qui effectue les inférences de sens.

Sur ce point, le regard de Toundi prend toute son importance. Sans que l'on ne sache vraiment si c'est la pratique scripturale elle-même qui influe sur sa manière de percevoir ou s'il s'agit d'une propension naturelle (comme il l'affirme), Toundi fouille du regard le champ de vision qui s'offre à lui, observant et notant chaque détail. Son champ de vision s'étend avec obstination. Il remarque tout, y compris ce qui relève en apparence du « superflu » ou du frivole, obligeant la décence à détourner les yeux : « [à l'église] Gosier-d'Oiseua profitait de l'élévation pour presser la main de sa voisine, tandis que les jambes de Mme Salvain se rapprochaient imperceptiblement de celles du commandant (54) ». Son journal se fait alors le réceptacle de cet excès, ou de cette violence du voir. Il acquiert, par là, une charge profondément ironique.

La peinture que Toundi fait de l'enterrement du père Gilbert (son protecteur) permet ainsi de souligner, indirectement, les enjeux de pouvoir et les déterminants sociaux qui conditionnent aussi bien le comportement des « Noirs » que celui de «Blancs ». Le comique se dégage du jeu 
mesuré des uns et des autres pour ne pas enfreindre les règles qui régissent l'attribution de l'espace. Là encore, il définit un espace de pouvoir, conditionné, territorialisé, afin de préserver la séparation idéologique des races :

Toute la Mission catholique Saint-Pierre de Dangan était là. [...] Le Blanc au long cou parla à l'un des gardes qui étaient dans sa voiture. Le garde alla compter dix pas en marchant contre la foule qui recula une, deux, trois... dix fois. Deux infirmiers transportèrent le corps du père Gilbert dans sa chambre. Les Blancs les suivirent. Le père Vandermayer les conduisit au salon. Quelques instants plus tard, il en ressortit, descendit l'escalier de quatre marches puis harangua la foule (2930).

De manière incidente, le texte signale la supercherie du discours idéologique français : il ne peut y avoir de « fraternité entre les races » dans un monde où les rapports reposent sur une alliance factice. Cette représentation d'un monde déshumanisé est renforcée par le caractère analytique des descriptions de Toundi («Les Blancs les suivirent. Le père Vandermayer les conduisit au salon $[\ldots] »)$, comme s'il voulait s'extraire de la dénomination pour toucher à une perception plus intuitive. Sous forme d'annotations, les éléments descriptifs tendent à briser l'harmonie de la représentation de façon à produire une impression de «mécanique plaqué sur du vivant», pour reprendre Henri Bergson (29). Le colon, à travers ses traits proéminents, devient ainsi une sorte d'obstacle monstrueux, presque antédiluvien : « Je me heurtai à un Blanc au long cou, puis à un autre, une espèce de masse ocre, qui me repoussèrent [...]» (Oyono 29). Le prêtre, dans son sermon, prend une allure affectée, jusqu'à l'obscénité : «Il fit un signe de croix et la foule l'imita. Il remonta l'escalier. A la dernière marche, ses mains descendirent sur ses fesses et lissèrent sa soutane (30)».

$\mathrm{Au}$ début du récit, Toundi n'a pourtant pas conscience de la charge subversive de son discours intimiste. Sa démarche d'écriture doit son origine au désir d'imiter son maître: «Maintenant que le révérend père Gilbert m'a dit que je sais lire et écrire couramment, je vais pouvoir tenir comme lui un journal. / - Je ne sais quel plaisir cache cette manière de Blanc, mais essayons toujours (15)». Du coup, le tableau qu'il brosse de son univers parait un jeu gratuit, comme si le journal était un sismographe qui enregistre mécaniquement les traces ou plutôt les symptômes d'une société coloniale sur le point de dépérir. A priori, il revient donc au lecteur la charge de décrypter les sinuosités de ce « langage du regard ».

Toutefois, progressivement et de manière latente, "l'écriture-observation " prend les accents d'une révolte. De même que le héros glisse subrepticement de l'identification aveugle au 
rejet dégoûté de ses modèles, son journal évolue du compte-rendu, de l'annotation systématique, au commentaire. Comme les points de suspensions qui parsèment le texte des cahiers le signalent typographiquement (suggérant par là une dynamique interprétative), le récit invite à dépasser le stade de la simple reconnaissance.

Mais cette prise de conscience s'embraye véritablement avec la découverte de la relation adultère de la femme du commandant, ainsi que l'épisode de la bastonnade, au cours duquel deux Africains soupçonnés de vol sont frappés à mort. Toundi, en effet, juge et interprète de plus en plus les faits et gestes de ses maîtres. Ce changement est reflété de manière incidente à travers les réactions de la femme du commandant: cette dernière ne supporte plus d'être observée par Toundi. Il est sa mauvaise conscience, «l'œil du sorcier » de Kalisia, non pas parce qu'il sait son comportement adultère, mais parce que son regard persistant pèse, à la manière d'un jugement muet : «Je baissai les yeux. Dans l'espace d'un éclair je les relevai. Ils rencontrèrent ceux de Madame. Je les vis devenir tout petits. Puis ils s'agrandirent comme si elle avait assisté à une scène d'épouvante (104)».

$\mathrm{Au}$ regard inquisiteur de Toundi correspond une écriture qui opère la déconstruction d'une lecture coloniale du monde, même si la rédaction du journal découle directement de celle-ci ${ }^{4}$. À la fin de la scène de la bastonnade, Toundi fait la remarque suivante : «Je pense à tous ces prêtres, ces pasteurs, tous ces Blancs qui veulent sauver nos âmes et qui nous prêchent l'amour du prochain. Le prochain du Blanc n'est-il que son congénère ? Je me demande, devant de pareilles atrocités, qui peut être assez sot pour croire à tous les boniments qu'on nous débite à l'Eglise et au Temple...» (115).

\section{« Vérité » du regard / artifice du discours}

Le texte oppose finalement l'ingénuité du regard du boy à l'artifice du discours impérialiste. Ses yeux perçant à nu, Toundi ébranle tout l'édifice idéologique des colons qui permettait d'asseoir leur domination, de faire prévaloir leurs privilèges et de justifier les exactions commises contre les «Noirs". Toutefois, gênés par son regard inquisiteur, les « Blancs » essayent de sauver la face en s'accrochant par tous les moyens à leurs faux-semblants, afin de maintenir une position privilégiée.

Dans son Portrait du colonisé, Albert Memmi dit : «Un effort constant du colonialiste consiste à expliquer, justifier et maintenir, par le verbe et la conduite, la place et le sort du 
colonisé, son partenaire dans le drame colonial et donc sa propre place (99) ». De même, les administrateurs européens, dans Une vie de boy, ripostent aux sous-entendus du regard de Toundi par un autre mode de communication allusive : un langage de la connivence. Mais d'une connivence précaire et artificielle, puisque elle va échouer.

La scène de la réunion dominicale du Cercle européen illustre bien cet aspect. À cette étape, le texte développe une construction alternée du discours mettant en regard un code verbal fondé sur l'hypocrisie et un langage visuel qui rapporte les faits et gestes des colons. Cette double caractérisation éclaire leur comportement infâme, mais aussi leur faillite à préserver les apparences. Dans cette séquence, Toundi, absorbé par le regard adultère de la femme du commandant, ne se rend pas compte qu'il verse trop de whisky aux colons qu'il doit servir. Profitant de cette aubaine, ceux-ci enchaînent un dialogue sur l'ivrognerie supposée des « Noirs », dressant ainsi un «portrait-accusation » du colonisé (Memmi 99). Prenant la parole à tour de rôle, chacun raconte une anecdote dans le but de conforter l'opinion selon laquelle tous les Africains sont par essence des alcooliques. Mais tandis que Toundi rapporte leurs propos désobligeants, il intègre, en incise, des observations sur leur comportement qui pourraient échapper à un œil inattentif. C'est alors en rétablissant le contexte d'énonciation, les circonstances de la parole, qu'il souligne tout l'artifice et les contradictions de leurs propos. Notamment, alors qu'ils pérorent sur l'ivresse des « Noirs », le journal laisse entrevoir que les colons eux-mêmes boivent sans arrêt. La réunion se termine dans l'ébriété générale : «Les Blancs vacillaient sur le plancher comme sur une peau de banane. Le commandant répondait par un grognement à tout ce qu'on lui disait » (Oyono 83).

D'autre part, le jeu mondain auquel ils s'adonnent en toute bonne conscience manque de finesse. Ils n'atteignent pas leur but parce qu'ils ne parviennent pas à susciter l'effet de surprise et de reconnaissance pourtant nécessaires à l'établissement d'une connivence. Une telle faillite de la collusion recherchée survient notamment lorsque Mme Salvain, la femme de l'instituteur, prend son tour de parole :

Mme Salvain toussa, comme pour se donner du courage. Toutes les têtes obliquèrent vers elle. Elle semblait avoir été oubliée avec son mari.

- Tous les matins, c'est d'abord l'odeur d'alcool et de crasse qui me parvient de la véranda. C'est ce qui m'annonce que mon boy est là...

Cette confidence n'eut aucun succès. M. Salvain leva les yeux au ciel. Un silence plana sur la salle. M. Janopoulos étouffa mal son hoquet avec une toux. Les Blancs manifestèrent ostensiblement qu'ils n'avaient rien remarqué (78). 
L'enchaînement des tours se fait par décrochements successifs, marqués par des silences prolongés et de reprises forcées. Ce «parasitage» du discours n'échappe pas à l'observation méthodique de Toundi, qui, par une espèce de «violence» muette, met en valeur en quoi les réactions des colons créent un effet de discordance avec ce qui vient d'être dit. Toundi détruit ainsi la portée des imputations coloniales qui ont servi à consolider un ordre social.

Cette coalition factice entre les « Blancs » est également métaphorisée par la réconciliation entre le commandant et sa femme, dont Toundi savait la relation adultère : "J'ai surpris le commandant et Madame en train de s'embrasser. Je croyais qu'il tiendrait plus longtemps. Il avait l'air d'un petit enfant qu'on surprend en train de voler ce qu'il a ostensiblement dédaigné. Après ça, je comprends que Madame puisse en faire tout ce qu'elle veut» (154). Comme l'a souligné Britwum, le regard de Toundi est un «regard remémorant» (38), mettant en relief les actions présentes du commandant à partir du passé de l'adultère. Gêné par cet « œil », les colons tentent alors de se débarrasser du boy en l'accusant à tort d'être le complice de Sophie, la femme de l'ingénieur, qui a quitté le pays en emportant la solde des ouvriers. Mais si Toundi n'échappe pas à la vengeance des colons, et meurt des séquelles d'un coup de crosse dans le thorax, son journal finit par le relayer, et devenir, à son tour, « l'œil du sorcier ».

\section{Conclusion}

L'enjeu de cette étude du regard dans Une vie de boy d'Oyono était de montrer comment le texte se situe à la jonction entre forme et signification, figure et réalité, texte et contexte, enfin, entre esthétique et représentation. Modalité allusive, énonciation implicite, forme sinueuse et insinuante, le regard, dans sa mise en scène romanesque, ne peut être saisi dans sa fixité. Il indique bien plus un mouvement, un discours possible, un horizon à définir. Il n'implique pas une reproduction fidèle du réel, ce qui conduirait à effacer le texte pour ne voir que sa référence, pas plus qu'il ne se donne pour un pamphlet anticolonial traduisant les vues de l'auteur. C'est dans la mesure où ce regard échappe, vagabonde, plus qu'il ne prend position, que le texte remonte à la surface, devient visible pour le lecteur et permet l'émergence d'une forme.

Ainsi, nous préférons nous garder des définitions du regard comme «instrument de témoignage » (Britwum), « camera cachée » (Amegbleame 158), ou encore de son assimilation, chez Bourgeacq, au miroir renversé du monde («looking-glass world ») ; expressions qui, en fin de compte, évacuent son caractère quasi «figural » (entendons par là ce qui est sensible, mais 
échappe au dit). En effet, il s'avère que le regard, chez Oyono, délimite une zone quelque peu floue : on l'a vu, Toundi transite entre l'observation naïve (une écriture intime, au fil des jours, sans direction précise) et une forme de révolte : son regard est un « regard-jugeant », déstabilisant et même menaçant.

Sans doute peut-on mettre en rapport le style d'Oyono avec sa trajectoire de diplomate (qui suivra l'entreprise d'écriture). L'auteur sait concilier les contraires pour mieux atteindre son but : montrer l'absurdité et la monstruosité d'un système impérialiste qui aliène autant le colonialiste que l'indigène, mais sans toutefois attaquer qui que ce soit, affichant par là son pacifisme ${ }^{6}$. C'est pourquoi il amène le lecteur à se laisser toucher par le propos plus qu'il ne cherche à la convaincre.

\section{Ouvrages cités}

Amegbleame, Simon. «Le regard du boy et la conscience du commandant : une lecture d'Une vie de boy de Ferdinand Oyono ». Le Blanc du Noir: Représentations de l'Europe et des Européens dans les littératures africaines. Dir. Susanne Gehrmann et János Riesz. Münster : LIT, 2004. 149-170.

Bergson, Henri. Le rire : Essai sur la signification du comique. Paris : PUF, 2004 [1940].

Bourgeacq, Jacques. «The Eye Motif and Narrative Strategy in Ferdinand Oyono's Une Vie de boy: An Ethno-Cultural Perspective ». The French Review. 66 : 5 (1993) : 788-799.

Britwum, Kwabena. 1977. «Regard, mémoire, témoignage : ou l'œil du sorcier dans Une vie de boy de Ferdinand Oyono ». Présence francophone 14 (1977) : 37-41.

Corcoran, Patrick. Une vie de boy and Le Vieux Nègre et la médaille. Londres : Grant \& Cutler, 2003.

Flannigan, Arthur. "'The Eye of the Witch' : Non-Verbal Communication and the Exercise of Power in Une Vie de Boy». The French Review $56: 1$ (1982) : 51-63.

Garnier, Xavier. «Les formes "dures" du récit: enjeux d'un combat». Notre librairie 148 (2002) : 54-58.

Mvomo Ela, Wullson. «Ferdinand Léopold Oyono, le haut responsable». Ecce Homo, Ferdinand Léopold Oyono: Hommage à un classique africain. Dir. Gervais Mendo Ze. Paris : Karthala, 2007. 19-41. 
Memmi, Albert. Portrait du colonisé : Précédé du Portrait du colonisateur et d'une préface de Jean-Paul Sartre. Paris : Payot, 1973 [1957].

Oyono, Ferdinand. Une vie de boy. Paris : Julliard, 1970 [1956].

Riffaterre, Michael. «L’illusion référentielle ». Littérature et réalité. Dir. Roland Barthes, Léo Bersani, Philippe Hamon, et al. Paris : Seuil, 1982. 91-118.

\footnotetext{
NOTES

${ }^{1}$ Oyono a été un témoin privilégié de cette situation. Son enfance fut marquée par la colonisation française, au Cameroun. Son père, notamment, était fonctionnaire au service de l'administration coloniale, avant d'être licencié à cause de la publication d'Une vie de boy. Mais c'est surtout la formation intellectuelle du jeune Oyono et son immigration à Paris qui vont lui permettre d'affiner sa perception de la réalité coloniale.

${ }^{2}$ Rappelons que, selon Michael Riffaterre (92), cette illusion renvoie à la croyance en un contact ou une relation directe entre mots et référents.

${ }^{3}$ Par exemple, on ne sait jamais ce qui, de la tentation du regard ou de l'exercice d'écriture, motive l'autre.

${ }_{5}^{4}$ Rappelons que Toundi écrit d'abord pour imiter son maître.

${ }^{5}$ Citant Peter Geschiere (Village Communities and the State, London, Kegan Paul, 1982, 101-104), Bourgeacq mentionne que « the djambe [associé au motif de l'œil] is a sort of looking-glass world, which looms behind every facet of social life (791). »

${ }^{6}$ Pour avoir un bon aperçu des prises de position politiques de Ferdinand Oyono, voir l'article de Wullsen Mvomo Ela (2007).
} 